\title{
Is futsal a donor sport for football?: exploiting complementarity for early diversification in talent development
}

\section{Bruno Travassos, Duarte Araújo \& Keith Davids}

To cite this article: Bruno Travassos, Duarte Araújo \& Keith Davids (2017): Is futsal a donor sport for football?: exploiting complementarity for early diversification in talent development, Science and Medicine in Football, DOI: 10.1080/24733938.2017.1390322

To link to this article: http://dx.doi.org/10.1080/24733938.2017.1390322

曲 Published online: 11 Oct 2017.

Submit your article to this journal $\llbracket$

a)

View related articles 주

View Crossmark data $₫$ 


\title{
Is futsal a donor sport for football?: exploiting complementarity for early diversification in talent development
}

\author{
Bruno Travassos (10), Duarte Araújo (10) ${ }^{\mathrm{b}}$ and Keith Davids (10) \\ aResearch Center in Sports Sciences, Health and Human Development, CIDESD, CreativeLab Research Community, Universidade da Beira Interior, \\ Covilhã, Portugal; bCIPER, Faculdade de Motricidade Humana, Universidade de Lisboa, Cruz Quebrada - Dafundo, Portugal; 'Centre for Sports \\ Engineering Research, Sheffield Hallam University, Sheffield, UK
}

\begin{abstract}
Introduction: In team sports like football, athlete development emerges through the continuous experience and practice of varied activities under variations in task and environmental constraints. Such variations in environmental and task constraints provide variable practice opportunities and experiences that promote an enrichment of the learning process through enhanced transfer, and the discovery of individual capabilities through diverse, functional play activities.

Objectives: In this commentary, we discuss theoretical insights that suggest how the sport of futsal can provide a useful basis for supporting the transfer of skills to performance in association football.

Conclusions: The complementary nature of the two sports can be exploited for skill acquisition in early diversification through emphasising selected performance-based affordances, behavioral correspondence between sports, and self-evident advances towards task goals. By taking up futsal at an early stage, future football players will have the opportunity to explore futsal tactical behaviors that will enrich their developing perceptual-motor landscape.

Practial Implications: To ensure a complementary transfer of capabilities between the sports, coaching interventions should highlight informational constraints to improve the coupling of perception and action in players in futsal and association football and promote the utilization of relevant affordances available in practice task designs.
\end{abstract}

\section{ARTICLE HISTORY}

Accepted 8 September 2017

\section{KEYWORDS}

Donor sport; transfer; expertise; basic movement skills

\section{Introduction}

Athlete development in sport emerges through the continuous interactions between an individual's personal capabilities and possibilities for action in performance environments (Davids and Baker 2007). Specifically, in team sports like football, it has been shown that the development of skill and expertise can be achieved through the experience and practice of varied activities under a variety of task and environmental constraints (Araújo et al. 2010). These constraints include playing football on the street, on the beach, on different fields with different formats, with or without goals and with different team conditions and rules (Araújo et al. 2010). Such variations in environmental and task constraints observed in Brazilian culture, for example, provide variable practice opportunities and experiences that promote an enrichment of the learning process through enhanced transfer, and the discovery of individual capabilities through diverse, functional play activities (Araújo et al. 2010). In this commentary, we discuss theoretical insights that suggest how the sport of futsal can provide a useful basis for supporting the transfer of skills to performance in association football. With reference to tenets of the Athletic Skills Model (ASM) (Wormhoudt et al. 2018), we outline, how the relationship between futsal and association football may be exploited, providing a conceptually and practically important for understanding athlete talent development in sports generally.
Previous research in many different sports has clarified how expert athletes tended to have taken part in a higher number of different sports as youngsters, whilst also experiencing a greater number of hours of practice in different sports than non-experts (Côté et al. 2007; Davids et al. 2017). These findings clearly support the proposal that early specialisation in sports practice and training in young children should be eschewed for an early diversification of experience in different physical activities. Research has pointed to the physical, psychological, emotional, and social problems, including dropout, which may result from early specialization. In contrast, from an ecological dynamics perspective, a more balanced experience, focused on early diversification, can enrich athletes' adaptive capacities in different domains that supports a possibility of transfer to specific performance contexts (Baker et al. 2009).

\section{Before specialising, the individual and the athlete}

It has been argued that early diversification experiences, in the form of participation in different sports, might strengthen the adaptive capacities of athletes by providing a platform for skilled performance in sport (Araújo and Davids 2011). A key question concerns how we may conceptualise and empirically verify the transfer of behaviors emerging under a particular set of task constraints to another set in elite sport performance contexts? In practical terms: how closely related or differentiated do 
different sports need to be to avoid early specialization issues and accrue early diversification benefits in development?

Prominent ideas in ecological dynamics propose that early practice and play experiences in children and developing athletes need to focus less attention on early specialization to avoid detrimental effects, and more attention on enhancing athleticism and general perceptual, cognitive and motor capacities. Based on these ideas, coaches need to understand when and how an athlete's practice task constraints can be modified to range between specific and general activities to encourage athleticism as a foundation for performance in a sport, even at more advanced learning stages. This type of practical intervention in coaching and learning designs requires a theoretically-supported notion of the concept of transfer, and how it may work in practice.

To verify the transfer of behaviors emerging under a particular set of task constraints to another set in elite sport performance contexts, in ecological dynamics some attempts have been made to evaluate and compare the emergent behaviors between practice tasks or between practice tasks and performance environment (Pinder et al. 2011; Travassos et al. 2012b). However, there is a clear need for studies in sport contexts which examine the practical utility of measuring transfer. But that is not an easy task, nor one that coaches and practitioners welcome due to the potential for disruptions to athlete performance in sport environments. Transfer needs to be carefully investigated in future through action-based research in sport performance environments, without affecting athlete behaviors through intrusions of manipulations. Ecological dynamics proposes that the evaluation of transfer could be based on the notion of action fidelity (i.e., the comparison of movement patterns between practice and performance contexts to identify more functional ones) (Stoffregen et al. 2003). Action fidelity should be viewed and assessed in relation to performance goal achievement, not considering "idealized" modes of acting. In line with this idea, the degree of performance goal achievement (e.g., in football, the number of successful passes completed or mean distance of shots to a target) could be considered as a measure of transfer. Ideas such as generality and specificity of transfer as advocated here, positive and negative transfer, positive and negative variability, need to be accounted for to capture action fidelity and how transfer of behaviors can occur between contexts of practice. General transfer occurs when an athlete undertakes nonspecific activities (playing different sports) which benefit the foundation of motor skills to perform in a target sport, by supporting adaptation of perception, decision-making, actions and cognitions during performance. Specificity of transfer is likely to emerge from "donor sports" or practising under specific task constraints of a target sport. Understanding positive and negative transfer effects in practice requires consideration of goal achievement and the positive (functional) or negative (nonfunctional) adaptation of players to constraints of specific performance environment. Related to the idea that movement variability can be positive or negative, there is a need to understand the activities that contribute to enhance performance functionality and those that decrease functionality. However, at this time there has been little research into this important topic. Theoretical work and empirical evidence is necessary to identify activities that could contribute to general or specific transfer in different sports. Regarding the important role of early diversification in the acquisition of skill, expertise and talent development, ecological dynamics is a theoretical model which can help us understand the intricate process of cross-fertilisation between sports. This is an important process that can provide a general development of athlete capacities and skills at an appropriate time in their careers, helping them to become better specialized athletes later, and avoiding some of the potential problems caused by premature specialization (e.g., see Araújo et al. 2010; Phillips et al. 2010; Bahr 2014). Early diversified practice and play in children and developing athletes can provide a behavioral foundation before talented athletes engage extensively in specialized practice, a significant amount of which can occur when the athlete is ready for it, physically, psychologically, socially, and emotionally (Davids et al. 2017).

These theoretical ideas are aligned with practitioner models of athlete development, such as the ASM, which clearly proposes how a dynamic transitioning can emerge between diverse sport experiences and specialization in a target sport (Wormhoudt et al. 2018). First and foremost, the ASM advocates the need to respect the development stages that individuals need to be exposed to in a variety of learning contexts that will help them explore functional movement solutions, while expanding their psychological, physical and physiological capabilities. The process of holistic development of individuals when interacting with different performance contexts contributes to improving the functional co-adaptation of individuals to performance environment constraints (Araújo et al. 2010). Also, the pleasure and fun associated with the constant discovery of new individual actions and possibilities for play in different performance contexts, increases their engagement with long-term motives for practice (Wormhoudt et al. 2018). Key ideas in ecological dynamics and the ASM point to a major problem with early specialization being an over-emphasis on specificity of transfer in practice at all levels, which is especially problematic in young children (Davids et al. 2017). While clearly important, there is a fundamental misconception that only specificity of transfer has utility in continuous athlete learning and development during practice. In this respect, the theory of ecological dynamics and the ASM argues that there needs to be a more nuanced understanding of the concept of transfer. Generality of transfer is useful at different times in practice, but especially early in learning to provide a foundation for more specialized experiences later in an athlete's development. Therefore, it is useful to consider transfer to exist on a continuum between high specificity and high generality (Davids et al. 2017). Consequently, a question of theoretical and practical importance is: how to use specificity and generality of transfer in sports practice and when?

\section{From general to specific transfer}

Whilst it is clear that specificity of transfer (predicated on specifying information from representative task constraints, fidelity of actions in practice and task goal achievement) is highly significant for the acquisition of skill and expertise in sport, its over-use and imposition very early in childhood does not come without some risks and challenges. The suggestion is that generality of transfer (predicated on non-specifying information, task constraints which 
may be varied and less representative of a specific performance context and involving actions which are not specific to particular sports) may be useful in the development of general processes and capacities which can be harnessed in later specialized training programmes (Davids et al. 2017; Wormhoudt et al. 2018). The ASM is particularly important in suggesting with some clarity how general capacities or movements skills can be transferred into five practical components: stability, flexibility, agility, power, and endurance. Also, the ASM suggests that the process of coordination can be subdivided in seven abilities: adaptability, coupling ability, ability to react, rhythmic ability, balance ability, spatial orientation ability and the kinetic differential ability (Wormhoudt et al. 2018). According to the ASM, such fundamental components of sport performance constitute the tools that each individual needs to have to develop skill, expertise and talent as an athlete.

It is now well established that the development of adaptive biological systems depends on the level of variability that such systems accommodate to face internal and external variations in performance conditions. In sport, increasing variability of practice promotes the exploration of new coordinative solutions through the exploitation of movement degrees of freedom to perform (Davids et al. 2003). It allows individuals to enhance their "dexterity" (Bernstein 1967) in becoming more flexible in re-organizing movement system degrees of freedom and exploring different information sources to satisfy changing task constraints (Chow 2013). Summarizing, variability of movement experiences and of practice in different contexts leads to the development of a broad foundation of perceptual, cognitive, social and movement skills (Côté et al. 2007). Thus, to promote general transfer, in early ages, children should experience a variety of physical activities and sports, even if the requisite movement patterns do not seem to have a specific relation with a specific target sport. Movement experiences could be designed to engage with open tasks in a range of environments, from more varied to more specific (Wormhoudt et al. 2018). The main idea is to develop more skilful individuals through exposure to a variety of non-specific and specific practice, allowing a more functional learning at the moment of specialization (Fransen et al. 2012). This process needs a careful and continuous transitioning between generality (non-target sports and activities) and specificity (engaging with various forms of a target sport) of transfer (Davids et al. 2017).

Engagement in the practice of complementary sports could contribute to develop early diversification with a focus on the holistic development of individuals that will support more specific transfer processes, ensuring a better (less detrimental) process of specialization in one specific sport. In the ASM (Wormhoudt et al. 2018) it has been proposed that some sports can act as "donor sports" which can benefit a transitioning phase between diversification and enhanced specialization of practice and training. Engagement with donor sports should be experienced especially before or at the beginning of the specialization phase in an athlete's development. For example, this proposition suggests that the sport of climbing can act as a donor sport to enhance performance in a target sport like gymnastics. The transfer may be enhanced due to similarities in the way that athletes in both sports need to use inter-limb coordinated actions for displacements in space and time, exploit externally available forces like momentum and friction, maintain equilibrium using one or more limbs on occasion, dynamically transfer weight, perceive information from a surface during engagement, and much more. Thus, the main issue for enhancing specificity of transfer is the exploitation of functional patterns of coordination in "donor sports", that are similar to the patterns of coordination required in a target sport. Furthermore, with regards to tactical behaviors in team games, transfer can be enhanced, by using "donor sports" that have similar perceptual-action requirements as in a target sport. These ideas suggest how, compared to sports in the very early diversification phase of development, donor sports should have a greater level of congruence and correspondence with target sports captured by specifying information available to regulate actions, representative tasks and action modes which are functional (related to goal attainment). Perception-action couplings developed through experience in a donor sport can be functionally relevant for supporting performance in a target sport, with practice and experience in both sports having mutually beneficial effects. This relationship can be harnessed during later specialized training while avoiding the documented risks associated with too much early specialization.

This theoretical rationale provides a principled basis to help coaches understand how they can explore and exploit the complementarity between sports, such as futsal and association football.

\section{Exploring the complementarity between "donor" sports}

The manipulation of practice task constraints or the variability of experience exploited in the practice of different sports could help individuals to strengthen the quality of the perception-action couplings that can be stabilised in learning, practice and experience to exploit sport affordances (i.e., action possibilities) during specialization phases. However, the same cannot be said for traditional practice methodologies which are over-focused on repetition and rehearsal of specific movement techniques (i.e., independently of the affordances that make these techniques functional) during structured drills in highly regulated practice conditions. Experience in donor sports (e.g., futsal in relation to association football) for athletes could facilitate the transitioning and exploitation of athletic abilities, based on how action can lead to perception, and how perception can guide action towards goal achievement in a target sport. This performance flexibility is predicated on system degeneracy, which is a key property for elite performance in competitive sport (Seifert et al. 2016).

To summarize, this is the fundamental theoretical conceptualization to explain how experience and practice in futsal can contribute to the development of skills in association football, just like association football can contribute to performance development in futsal. The complementary relations between different sports could support coaching interventions to design practice tasks that highlight informational constraints that promote exploration, discovery, and adaptations in learners.

What could these theoretical ideas imply in the futsalassociation football complementary relationship? Futsal is a 5 -a-side game played indoors in a $40 \mathrm{~m} \times 20 \mathrm{~m}$ playing area $\left(80 \mathrm{~m}^{2}\right.$ 
of area per player). It is an intense sport which requires constant changes of direction, accelerations and decelerations, quick and precise tactical and technical actions with and without the ball to successfully perform (Castagna et al. 2009). The restricted variations in space for performing individual actions, as well as the variations in offensive and defensive collective playing systems, offer a great opportunity to players to improve individual technical and tactical capabilities, but also the management of space from a collective perspective (Travassos et al. 2012a).

In contrast, association football is an 11-a-side game played in a $90 \mathrm{~m} \times 120 \mathrm{~m}$ playing area $\left(490 \mathrm{~m}^{2}\right.$ of area per player). Football is characterized by intermittent actions, combining periods of maximal/near maximal efforts with unpredictable movement patterns and explosive technical actions (Mohr et al. 2003; Di Salvo et al. 2007). The large space for action and the number of players involved in the game leads to players spending large periods of the game without the ball in physical states of lower intensity and engaged in fewer decisional activities, in contrast to less frequent periods spent performing high intensity activities when near or in possession of the ball (Di Salvo et al. 2009).

Previous studies have highlighted that individual and collective tactical actions in futsal (Corrêa et al. 2012; Travassos et al. 2016) and association football (Lago et al. 2010; Duarte et al. 2012; Sampaio et al. 2014) are context-dependent. In this sense, ball management and general movement patterns are information-regulated in both sports. However, due to the number of players involved, and especially the space and time available for playing, futsal requires precise technical and tactical actions with and without the ball. In contrast, football requires high intensity activities with and without the ball, but with more time and space to perform these actions in comparison with futsal.

Highlighting similar basic movements comparing futsal with association football, due to the small space available for play, futsal's main emphasis is on ball control and manipulation, taking different types of touches of the ball in tight spaces (i.e. using "soft feet" to gently manipulate the ball in small spaces), using different parts of the feet (such as the sole, the sides, back and toe) and timing to pass, shoot and dribble the ball (which is smaller (size 3), and has a lower coefficient of restitution than a regulation size 5 football ball) (Araújo et al. 2004). In contrast, in football the emphasis is on performance of gross movements, due to higher space and time to perform, requiring more strength and explosive power. Associated with the need to move the ball quickly and with precision, or to maintain defensive equilibrium to recover ball possession, in line with the ASM proposals, futsal promotes general individual agility, in terms of coordination, coupling ability, ability to react, rhythmic ability, and balance ability.

From a collective system perspective, futsal requires equilibrium in defensive and offensive moves with precise adjustments according to variations in the space-time interrelations between teammates and opponents, promoting the development of players' spatial orientation and the ability to functionally manage space and time during performance. The constant changes in players' positioning increases variability in space covered and in relationships established with teammates and opponents, providing a broader perception of game relations.
Additionally, the fewer number of players constituting a futsal team, in comparison with football, contributes to the development of a wide range of technical and tactical abilities. Futsal also provide more frequent opportunities to perform skills and engage with the ball, compared to football (see Fenoglio 2003; Davids et al. 2013). Every player is required to use both feet to perform all the skills needed during competitive performance, as well as engage in collective tactical behaviors, with and without the ball. Such a reduction in the number of players in futsal decreases the complexity of the game, compared to association football, focusing players on available possibilities for action in a narrower field of affordances during performance. Based on the notion of affordances (opportunities for action) ecological dynamics, suggests that futsal can help football players to explore local affordances in an affordance landscape (Davids et al. 2017). That is, to discover, explore and exploit information and possibilities for action that can help players manipulate the ball in tight spaces to move the ball past opponent and create instabilities in defensive lines (Lopez-Felip and Turvey 2017).

Furthermore, futsal could be a better option to promote transfer effects in practice than small-sided football games (e.g., 4v4), due to the different perceptual-motor adaptations of actions required because of the type of ball used, the characteristics of futsal court surfaces and the even the type of futsal shoes used. Also, by taking up futsal at an early stage, future football players will have the opportunity to explore different offensive and defensive tactical behaviors, founded on those in futsal, that will enrich their developing perceptual-motor landscape. This landscape can provide a resource of developing movement patterns and behaviors that player can exploit when seeking to enhance transfer between donor and target sports.

\section{Practical Implications}

To ensure a complementary transfer of capabilities between the sports, coaching interventions should highlight informational constraints to improve the coupling of perception and action in players in futsal and association football and promote the utilization of relevant affordances available in practice task designs. The development of such complementarities cannot be based on practice tasks that promote repetition of structured drills or pre-determined practice tasks (Araújo and Davids 2015). Rather the complementary nature of the two sports can be exploited for skill acquisition in early diversification through emphasising selected performance-based affordances, behavioral correspondence between sports, and self-evident advances towards task goals.

\section{Acknowledgments}

This work was partly supported under Grants FCT (UID/DTP/04045/2013) and COMPETE (POCI-01-0145-FEDER-006969) to CIDESD - Research Center in Sport, Health and Human Development and FCT (UID/DTP/UI447/2013) to CIPER - Centro Interdisciplinar para o Estudo da Performance Humana. 


\section{Disclosure statement}

No potential conflict of interest was reported by the authors.

\section{Funding}

This work was supported by the Fundação para a Ciência e Tecnologia [UID/ DTP/04045/2013,UID/DTP/UI447/2013]; COMPETE [POCI-01-0145-FEDER $-006969]$.

\section{ORCID}

Bruno Travassos (iD http://orcid.org/0000-0002-2165-2687

Duarte Araújo (D) http://orcid.org/0000-0001-7932-3192

Keith Davids (D) http://orcid.org/0000-0003-1398-6123

\section{References}

Araújo D, Davids K. 2011. What exactly is acquired during skill acquisition? J Conscious Stud. 18(4):7-23.

Araújo D, Davids K. 2015. Towards a theoretically-driven model of correspondence between behaviours in one context to another: implications for studying sport performance. Int J Sport Pshychol. 46(6):266-280.

Araújo D, Davids K, Bennett S, Button C. 2004. Emergence of sport skills under constraints. In: Williams AM, Hodges NJ, editors. Skill acquisition in sport: research, theory and practice. London: Routledge, Taylor \& Francis; p. 409-433.

Araújo D, Fonseca C, Davids K, Garganta J, Volossovitch A, Brandão R, Krebs R. 2010. The role of ecological constraints on expertise development. Talent Dev Excell. 2(2):165-179.

Bahr R. 2014. Demise of the fittest: are we destroying our biggest talents? Br J Sports Med. 48(17):1265-1267.

Baker J, Cobley S, Fraser-Thomas J. 2009. What do we know about early sport specialization? Not much! High Ability Studies. 20(1):77-89.

Bernstein N. 1967. The co-ordination and regulation of movements. Oxford: Pergamon Press.

Castagna C, D'Ottavio S, Granda-Vera J, Barbero-Alvarez JC. 2009. Match demands of professional Futsal: a case study. J Sci Med Sport. 12(4):490-494.

Chow JY. 2013. Nonlinear learning underpinning pedagogy: evidence, challenges, and implications. Quest. 65(4):469-484.

Corrêa U, Alegre F, Freudenheim A, Dos Santos S, Tani G. 2012. The game of futsal as an adaptive process. Nonlinear Dynamics Psychol Life Sci. 16(2):185.

Côté J, Baker J, Abernethy B. 2007. Practice and play in the development of sport expertise. Handbook Sport Psychol. 3:184-202.

Davids K, Araújo D, Correia V, Vilar L. 2013. How small-sided and conditioned games enhance acquisition of movement and decision-making skills. Exerc Sport Sci Rev. 41(3):154-161.

Davids K, Baker J. 2007. Genes, environment and sport performance: why the nature-nurture dualism is no longer relevant. Sports Med. 37(11):961-980.

Davids K, Glazier P, Araújo D, Bartlett R. 2003. Movement systems as dynamical systems: the functional role of variability and its implications for sports medicine. Sports Med. 33(4):245-260.

Davids K, Güllich A, Araújo D, Shuttleworth R. 2017. Understanding environmental and task constraints on athlete development: analysis of micro-structure of practice and macro-structure of development histories. In: Baker, J, Cobley, S, Schorer, J, Wattie, N, editors. Routledge handbook of talent identification and development in sport. London: Routledge; p. 192-206.

Di Salvo V, Baron R, Tschan H, Montero F, Bachl N, Pigozzi F. 2007. Performance characteristics according to playing position in elite soccer. Int J Sports Med. 28(3):222-227.

Di Salvo V, Gregson W, Atkinson G, Tordoff P, Drust B. 2009. Analysis of high intensity activity in Premier League soccer. Int J Sports Med. 30 (03):205-212.

Duarte R, Araújo D, Freire L, Folgado H, Fernandes O, Davids K. 2012. Intraand inter-group coordination patterns reveal collective behaviours of football players near the scoring zone. Hum Mov Sci. 31(6):1639-1651.

Fenoglio R. 2003. The Manchester United 4 V 4 pilot scheme for under 0's: part II - the analysis. Insight: Football Assoc Coaches Magaz. 6(4):21-24.

Fransen J, Pion J, Vandendriessche J, Vandorpe B, Vaeyens R, Lenoir M, Philippaerts RM. 2012. Differences in physical fitness and gross motor coordination in boys aged 6-12 years specializing in one versus sampling more than one sport. J Sports Sci. 30(4):379-386.

Lago C, Casais L, Dominguez E, Sampaio J. 2010. The effects of situational variables on distance covered at various speeds in elite soccer. Eur J Sport Sci. 10(2):103-109.

Lopez-Felip MA, Turvey MT. 2017. Desideratum for GUT: A functional semantics for sport. Hum Mov Sci. doi:10.1016/j.humov.2017.05.002

Mohr M, Krustrup P, Bangsbo J. 2003. Match performance of high-standard soccer players with special reference to development of fatigue. J Sports Sci. 21(7):519-528.

Phillips E, Davids K, Renshaw I, Portus M. 2010. Expert performance in sport and the dynamics of talent development. Sports Med. 40(4):271-283.

Pinder R, Renshaw I, Davids K, Kerhervé H. 2011. Principles for the use of ball projection machines in elite and developmental sport programmes. Sports Med. 41(10):793-800.

Sampaio J, Lago C, Gonçalves B, Maçãs V, Leite N. 2014. Effects of pacing, status and unbalance in time motion variables, heart rate and tactical behaviour when playing 5 -a-side football small-sided games. J Sci Med Sport. 17(2):229-233.

Seifert L, Komar J, Araújo D, Davids K. 2016. Neurobiological degeneracy: a key property for functional adaptations of perception and action to constraints. Neurosci Biobehavioral Rev. 69:159-165.

Stoffregen T, Bardy B, Smart L, Pagulayan R. 2003. On the nature and evaluation of fidelity in virtual environments. In: Hettinger $\sqcup$, Haas MW, editors. Virtual and adaptive environments: applications, implications, and human performance issues. Mahwah (NJ): Lawrence Erlbaum Associates; p. 111-128.

Travassos B, Araújo D, Duarte R, McGarry T. 2012a. Spatiotemporal coordination behaviors in futsal (indoor football) are guided by informational game constraints. Hum Mov Sci. 31(4):932-945.

Travassos B, Bourbousson J, Esteves P, Marcelino R, Pacheco M, Davids K. 2016. Adaptive behaviours of attacking futsal teams to opposition defensive formations. Hum Mov Sci. 47:98-105.

Travassos B, Duarte R, Vilar L, Davids K, Araújo D. 2012b. Practice task design in team sports: representativeness enhanced by increasing opportunities for action. J Sports Sci. 30(13):1447-1454.

Wormhoudt R, Savelsbergh GJP, Teunissen JW, Davids K. 2018. Athletics Skills Model for optimizing talent development through movement education: no specialists, but athletes with a specialization: A new avenue to think about movement. London: Routledge. 